\title{
The effect of fluid overload on sleep apnoea severity in haemodialysis patients
}

\author{
Owen D. Lyons ${ }^{1,2,3}$, Toru Inami ${ }^{1,2}$, Elisa Perger ${ }^{1,2}$, Azadeh Yadollahi ${ }^{1}$, \\ Christopher T. Chan ${ }^{4}$ and T. Douglas Bradley ${ }^{1,2,4}$
}

Affiliations: 'Sleep Research Laboratory, University Health Network Toronto Rehabilitation Institute, Toronto, ON, Canada. ${ }^{2}$ Centre for Sleep Medicine and Circadian Biology, University of Toronto, Toronto, ON, Canada. ${ }^{3}$ Dept of Medicine, Women's College Hospital, Toronto, ON, Canada. ${ }^{4}$ Dept of Medicine, University Health Network Toronto General Hospital, Toronto, ON, Canada.

Correspondence: T. Douglas Bradley, University Health Network Toronto General Hospital, 9N-943, 200 Elizabeth St, Toronto, ON, M5G 2C4, Canada. E-mail: douglas.bradley@utoronto.ca

@ERSpublications

Fluid overload plays a key role in the pathogenesis of OSA and CSA in end-stage renal disease http://ow.ly/zSZo308a8TP

Cite this article as: Lyons OD, Inami T, Perger E, et al. The effect of fluid overload on sleep apnoea severity in haemodialysis patients. Eur Respir J 2017; 49: 1601789 [https://doi.org/10.1183/13993003.017892016].

ABSTRACT As in heart failure, obstructive and central sleep apnoea (OSA and CSA, respectively) are common in end-stage renal disease. Fluid overload characterises end-stage renal disease and heart failure, and in heart failure plays a role in the pathogenesis of OSA and CSA. We postulated that in end-stage renal disease patients, those with sleep apnoea would have greater fluid volume overload than those without.

End-stage renal disease patients on thrice-weekly haemodialysis underwent overnight polysomnography on a nondialysis day to determine their apnoea-hypopnoea index (AHI). Extracellular fluid volume of the total body, neck, thorax and right leg were measured using bioelectrical impedance.

28 patients had an AHI $\geqslant 15$ (sleep apnoea group; OSA:CSA 21:7) and 12 had an AHI $<15$ (no sleep apnoea group). Total body extracellular fluid volume was $2.6 \mathrm{~L}$ greater in the sleep apnoea group than in the no sleep apnoea group $(\mathrm{p}=0.006)$. Neck, thorax, and leg fluid volumes were also greater in the sleep apnoea than the no sleep apnoea group $(\mathrm{p}<0.05)$, despite no difference in body mass index $(\mathrm{p}=0.165)$.

These findings support a role for fluid overload in the pathogenesis of both OSA and CSA in end-stage renal disease.

Earn CME accreditation by answering questions about this article. You will find these at erj.ersjournals.com/journal/cme Received: Sept 082016 | Accepted after revision: Jan 052017

Support statement: Operating grant MOP-82731 from the Canadian Institutes of Health Research. O.D. Lyons was supported by a joint Canadian Thoracic Society/European Respiratory Society Peter Macklem Research Fellowship and the Joseph M. West Family Memorial Fund Postgraduate Research Award, T. Inami by an unrestricted fellowship from Philips Respironics (Japan), C.T. Chan by the R. Fraser Elliot Chair in Home Dialysis, A. Yadollahi by fellowships from the Toronto Rehabilitation Institute, Mitacs Elevate programme and a CIHR Training Grant in Sleep and Biological Rhythms, and T.D. Bradley by the Clifford Nordal Chair in Sleep Apnoea and Rehabilitation Research, and the Godfrey S. Pettit Chair in Respiratory Medicine. Funding information for this article has been deposited with the Open Funder Registry.

Conflict of interest: None declared.

Copyright OERS 2017 


\section{Introduction}

Obstructive sleep apnoea (OSA) is common in the general population, having a prevalence of $3-17 \%$, while central sleep apnoea (CSA) is rare, with a prevalence of $<1 \%[1,2]$. However, among patients with fluid overload states, such as end-stage renal disease (ESRD), the overall prevalence of sleep apnoea is much higher, at $\sim 50-60 \%$ [3-6], similar to that in heart failure [7], another condition characterised by fluid overload. The increased prevalence of sleep apnoea in both these conditions is not explained by age or body mass index (BMI) [8-10], suggesting that fluid overload itself may play a role in the pathogenesis of both OSA and CSA in ESRD and heart failure.

Fluid overload is an independent predictor of increased mortality in ESRD [11]. The presence of sleep apnoea in ESRD is also associated with increased mortality [12]. This raises the possibility that fluid overload may contribute to increased mortality at least partially through its effect on sleep apnoea. Accordingly, a better understanding of the mechanisms that contribute to the pathogenesis of sleep apnoea in ESRD could facilitate the application of therapies other than continuous positive airway pressure, such as more aggressive fluid volume control, with the aim of reducing morbidity and mortality in this at-risk population.

It has been shown that intensification of dialysis, by conversion from thrice weekly conventional haemodialysis to nocturnal haemodialysis 6 nights per week led to attenuation of sleep apnoea. However, the contribution of attenuation of uraemia versus reduced fluid volume on this effect was not elucidated [13]. To this end, we recently showed in ESRD patients on conventional haemodialysis with either OSA or CSA that removal of $2.2 \mathrm{~L}$ of fluid, in a single session of ultrafiltration, led to a $36 \%$ reduction in the frequency of apnoea and hypopnoea events per hour of sleep (apnoea-hypopnoea index (AHI)) [14] in the absence of any change in uraemia or acid-base status. Conversely, we showed that infusion of $\sim 2 \mathrm{~L}$ of saline at sleep onset led to a three-fold increase in the AHI in a group of nonobese healthy older males [15]. Taken together, these findings suggest that fluid overload contributes to the pathogenesis of OSA and CSA [16-19]. Accordingly, we hypothesised that in ESRD patients, those with sleep apnoea would have higher total body, leg, thoracic and neck fluid volumes than those without sleep apnoea.

\section{Methods}

Subjects

Inclusion criteria were patients with ESRD aged $\geqslant 18$ years undergoing conventional thrice weekly haemodialysis in the University Health Network (Toronto, ON, Canada), all of whom underwent annual echocardiography. Patients were recruited consecutively irrespective of symptoms of sleep apnoea. Exclusion criteria were patients who were already treated for sleep apnoea, had a BMI $>35 \mathrm{~kg} \cdot \mathrm{m}^{-2}$, tonsillar hypertrophy or a left ventricular ejection fraction $<45 \%$ by echocardiography (figure 1 ). The protocol was approved by the research ethics board of the University Health Network and all subjects provided written informed consent before participation.

\section{Polysomnography}

All subjects underwent overnight polysomnography (PSG). Prior to PSG, demographic characteristics, medical history and prescribed medications were recorded. PSG was performed using standard techniques and scoring criteria for sleep stages, arousals from sleep and periodic leg movements (PLM), by personnel blind to fluid volume measurements [20,21]. All subjects slept on a single pillow with the bed flat. Body position was determined by video monitoring. Thoracoabdominal motion was monitored by respiratory inductance plethysmography, and nasal airflow by nasal pressure cannulae (BiNAPS model 5500; Salter Labs, Arvin, CA, USA). Arterial oxyhaemoglobin saturation was monitored by oximetry. Central apnoea was

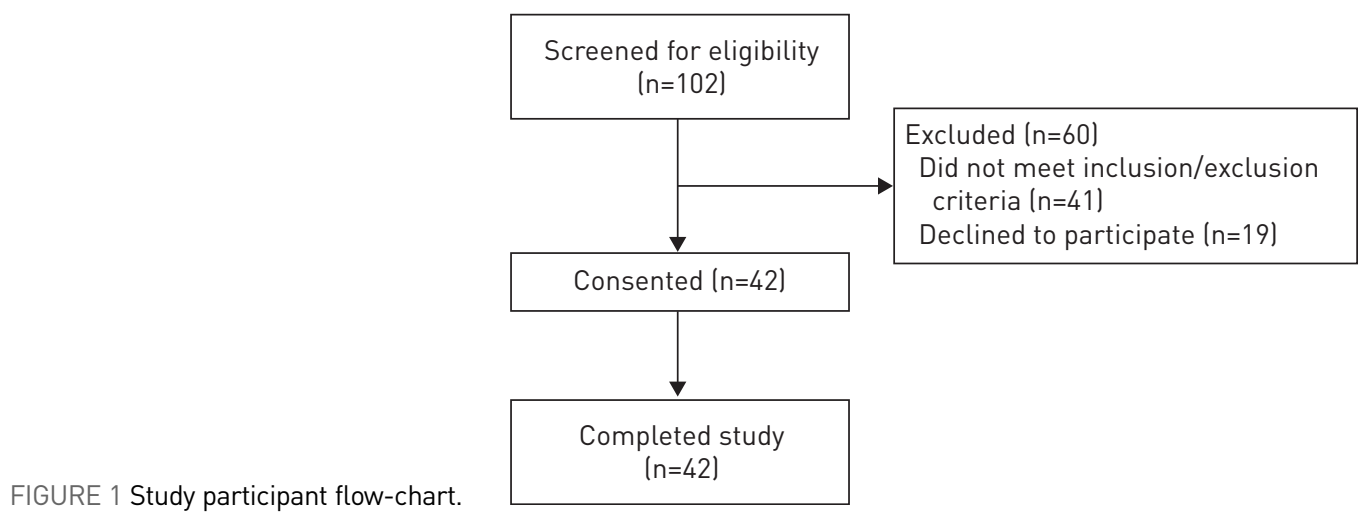


defined as $\geqslant 90 \%$ reduction in tidal volume for $\geqslant 10 \mathrm{~s}$ in which thoracoabdominal motion was absent, and central hypopnoea as a reduction of $\geqslant 30 \%$ in tidal volume for $\geqslant 10 \mathrm{~s}$ with in-phase thoracoabdominal motion without airflow limitation on nasal pressure accompanied by a $\geqslant 3 \%$ desaturation or an arousal. Obstructive apnoeas and hypopnoeas were similarly defined, except that they had to be accompanied by out-of-phase thoracoabdominal motion or airflow limitation on nasal pressure, respectively [21]. Subjects were divided into a no sleep apnoea group $(\mathrm{AHI}<15)$ and a sleep apnoea group $(\mathrm{AHI} \geqslant 15)$. Subjects with sleep apnoea were subdivided into those with OSA, in whom $>50 \%$ of events were obstructive, and CSA, in whom $>50 \%$ of events were central. Signals were recorded on a computerised sleep recording system (Sandman; Nellcor Puritan Bennett, Ottawa, ON, Canada) and scored by technicians blind to fluid volume measurements.

\section{Fluid volumes}

Height and body weight were measured before going to bed and on awakening the next morning. With subjects instrumented for PSG, lying awake and supine, total body fluid volume, intracellular fluid volume and extracellular fluid volume (ECFV) were assessed by measuring the impedance to electrical flow between electrodes placed on the right ankle and the right hand using a bioelectrical impedance device (Xitron Hydra, model 4200; Xitron Technologies, San Diego, CA, USA). This well-validated technique uses impedance to electrical current within a body segment to measure fluid content [22]. ECFVs of leg (LECFV), thorax (TECFV) and neck (NECFV) were measured simultaneously using another bioelectrical impedance device (MP150; Biopac Systems Canada, Montreal, QC, Canada). This device uses multifrequency signalling to allow for simultaneous recording of the impedance in multiple body segments [23]. Sensing electrodes were placed on the ankle and upper thigh of the right leg for LECFV; for TECFV, they were positioned on the midline of the posterior aspect of the chest: one at the superior border of the scapula and one at the same level as the xiphoid process; and for NECFV, on the right side of the neck: one below the right ear and one at the base of the neck. Measurements of total body fluid, total body ECFV, LECFV, TECFV and NECFV were repeated the next morning after awakening and before subjects got out of bed and the overnight changes calculated. Measured fluid volumes were adjusted for body surface area (fluid volume index).

\section{Statistical analysis}

Continuous variables were expressed as mean $\pm \mathrm{SD}$ and categorical variables as proportions. Differences in measurements between the two groups were analysed using t-tests or Wilcoxon tests for normally and non-normally distributed variables, respectively. Relationships between independent variables and the presence of sleep apnoea were examined by logistic regression. The independent factors included in the logistic regression were determined by whether they were found to be significantly different between groups. Univariate and multivariate linear regression analyses were performed with AHI as the dependent variable. A two tailed p-value $<0.05$ was considered significant. Analyses were performed using SPSS (23.0.1; SPSS, Chicago, IL, USA).

\section{Results}

\section{Subjects}

42 patients were recruited and underwent a baseline PSG. Of the 42, 28 had an AHI $\geqslant 15$ (21 OSA, seven CSA). The baseline characteristics of all 42 subjects are shown in table 1 . The sleep apnoea group was older and had a higher proportion of males than the no sleep apnoea group. There was no difference in

TABLE 1 Baseline characteristics of the subjects

\begin{tabular}{|c|c|c|c|}
\hline & $\begin{array}{c}\text { No sleep apnoea } \\
A H I<15\end{array}$ & $\begin{array}{c}\text { Sleep apnoea } \\
A H I \geqslant 15\end{array}$ & p-value \\
\hline Subjects & 14 & $28^{\#}$ & \\
\hline AHI events $\cdot h^{-1}$ & $5.2 \pm 3.1$ & $43.9 \pm 25.2$ & $<0.0001$ \\
\hline Age years & $36.4 \pm 11.5$ & $52.9 \pm 11.2$ & $<0.0001$ \\
\hline Male:female & $6: 8$ & $22: 6$ & 0.021 \\
\hline BMI $\mathrm{kg} \cdot \mathrm{m}^{-2}$ & $22.9 \pm 4.1$ & $24.3 \pm 4.4$ & 0.165 \\
\hline Left ventricular ejection fraction \% & $62.9 \pm 4.2$ & $61.2 \pm 4.0$ & 0.546 \\
\hline Hypertension & $12(85.7)$ & $26(92.8)$ & 0.590 \\
\hline Ischaemic heart disease & $1(7.1)$ & $2(7.1)$ & 1.000 \\
\hline Atrial fibrillation & $0(0.0)$ & $1(3.6)$ & 1.000 \\
\hline Diabetes & $2(14.3)$ & $5(17.9)$ & 1.000 \\
\hline
\end{tabular}

Data are presented as $n$, mean \pm SD or $n(\%)$, unless otherwise stated. AHI: apnoea-hypopnoea index; BMI: body mass index. ${ }^{\#}$ : obstructive sleep apnoea $n=21$, central sleep apnoea $n=7$. 
BMI between the groups. Of the 42 patients, 17 (40.5\%) were on an angiotensin converting enzyme inhibitor and/or an angiotensin receptor blocker, 23 (55\%) were on a $\beta$-blocker, 15 (35.7\%) were on a calcium-channel antagonist and three (7.1\%) were on a low dose of prednisone. None of the patients was on any sedative medication or diuretic. All were receiving adequate dialysis, as indicated by a percentage reduction of urea $>65 \%$ post-dialysis (table 2 ).

\section{Sleep structure}

Sleep structure of the two groups is shown in table 3. Compared to the no sleep apnoea group, the sleep apnoea group had significantly less slow wave sleep $(\mathrm{p}=0.001)$ and a higher arousal index $(\mathrm{p}=0.001)$. There was no difference in supine sleep time $(\mathrm{p}=0.321)$ or the PLM index $(\mathrm{p}=0.535)$ between the groups.

\section{Fluid volumes}

As displayed in figure 2, compared to the no sleep apnoea group, the sleep apnoea group had higher total body ECFV ( $\mathrm{p}=0.006)$. In addition, as shown in table 4, the sleep apnoea group had higher evening LECFV ( $p=0.010)$, evening TECFV $(p=0.028)$ and evening NECFV $(p=0.016)$. These differences persisted after normalising for body surface area. In the sleep apnoea group there was a greater overnight reduction in LECFV ( $p=0.048)$, compared to the no sleep apnoea group, and a trend towards a greater overnight change in TECFV that did not reach statistical significance $(p=0.059)$. There was no difference in the overnight change in NECFV between the groups $(\mathrm{p}=0.430)$.

\section{Relationships between independent variables and the presence of sleep apnoea}

In a multiple logistic regression analysis, with the presence of sleep apnoea as the dependent variable, and age, sex and total body ECFV index as independent variables, the only variables independently associated with the presence of sleep apnoea were age $(\mathrm{p}=0.005)$ and total body ECFV index $(\mathrm{p}=0.047)$ (table 5).

\section{Relationships between independent variables and AHI}

In univariate regression analyses, there were significant correlations between AHI and male sex $(r=0.368$, $\mathrm{p}=0.019)$ and total body ECFV index $(\mathrm{r}=0.468, \mathrm{p}=0.002)$. There was no relationship between AHI and age $(\mathrm{r}=0.263, \mathrm{p}=0.093)$ or BMI $(\mathrm{r}=0.162, \mathrm{p}=0.306)$. In a multivariable linear regression analysis with AHI as the dependent variable and age, BMI, sex and total body ECFV index as independent variables, total body ECFV index was the only independent correlate of the AHI $(r=0.468, p=0.002)$.

\section{Discussion}

The key finding of this study was that ESRD patients with sleep apnoea had a mean total body ECFV $2.6 \mathrm{~L}$ greater than those without sleep apnoea. While there was a higher proportion of males and older subjects in the sleep apnoea group compared to the no sleep apnoea group, there was no difference in BMI between the groups. Age and total body ECFV index were the only independent factors associated with the presence of sleep apnoea, and furthermore, total body ECFV index was the only factor that correlated independently with sleep apnoea severity as assessed by the AHI. Another important finding in this study was that the sleep apnoea group had higher LECFV, TECFV and NECFV in the evening, and a greater overnight reduction in LECFV than the no sleep apnoea group. These results are in keeping with evidence that fluid overload is an important factor in the pathogenesis of sleep apnoea in chronic kidney disease and in ESRD.

\begin{tabular}{|c|c|c|c|}
\hline & $\begin{array}{c}\text { No sleep apnoea } \\
A H I<15\end{array}$ & $\begin{array}{c}\text { Sleep apnoea } \\
A H I \geqslant 15\end{array}$ & p-value \\
\hline Subjects & 14 & 28 & \\
\hline Treatment duration $\mathrm{h}$ & $4 \pm 0$ & $4 \pm 0$ & 1.000 \\
\hline Frequency sessions-week ${ }^{-1}$ & $3 \pm 0$ & $3 \pm 0$ & 1.000 \\
\hline $\mathrm{PRU}^{\#} \%$ & $74.4 \pm 5.9$ & $73.5 \pm 7.1$ & 0.713 \\
\hline$K t / V^{\#}$ & $1.6 \pm 0.4$ & $1.6 \pm 0.4$ & 0.725 \\
\hline
\end{tabular}

Data are presented as $n$ or mean $\pm S D$, unless otherwise stated. AHI: apnoea-hypopnoea index; PRU: percentage reduction in urea; $K$ : dialyser clearance of urea; $t$ : dialysis time; $V$ : volume of distribution of urea. ": PRU and $K t / V$ are commonly used clinical measures of adequacy of dialysis. PRU is calculated from the pre- and post-dialysis urea levels; a PRU $>65 \%$ is considered to indicate adequate dialysis. In general, a $K t / V>1.2$ is considered to indicate adequate dialysis. 
TABLE 3 Sleep structure

\begin{tabular}{|c|c|c|c|}
\hline & $\begin{array}{c}\text { No sleep apnoea } \\
A H I<15\end{array}$ & $\begin{array}{c}\text { Sleep apnoea } \\
A H I \geqslant 15\end{array}$ & p-value \\
\hline Subjects & 14 & 28 & \\
\hline TST h & $4.7 \pm 0.8$ & $4.4 \pm 1.4$ & 0.702 \\
\hline Sleep efficiency \% & $81 \pm 12$ & $70 \pm 21$ & 0.155 \\
\hline Slow-wave sleep min & $59 \pm 34$ & $22 \pm 23$ & 0.001 \\
\hline Slow-wave sleep $\%$ of TST & $20 \pm 10$ & $8 \pm 8$ & 0.001 \\
\hline REM sleep min & $53 \pm 18$ & $42 \pm 27$ & 0.104 \\
\hline REM sleep \% of TST & $18 \pm 7$ & $14 \pm 8$ & 0.114 \\
\hline Arousal index events $\cdot \mathrm{h}^{-1}$ & $22 \pm 12$ & $49 \pm 30$ & 0.001 \\
\hline PLM index events $\cdot \mathrm{h}^{-1}$ & $16 \pm 24$ & $25 \pm 31$ & 0.535 \\
\hline Minimum $\mathrm{SaO}_{2} \%$ & $92 \pm 3$ & $83 \pm 16$ & $<0.001$ \\
\hline Transcutaneous $\mathrm{PCO}_{2} \mathrm{mmHg}$ & $39.5 \pm 4.2^{\#}$ & $39.6 \pm 3.2^{9}$ & 0.664 \\
\hline Supine time $h$ & $2.6 \pm 1.8$ & $2.0 \pm 1.9$ & 0.321 \\
\hline
\end{tabular}

Data are presented as $\mathrm{n}$ or mean $\pm \mathrm{SD}$, unless otherwise stated. AHI: apnoea-hypopnoea index; TST: total sleep time; REM: rapid eye movement; PLM: periodic leg movement; $\mathrm{SaO}_{2}$ : arterial oxygen saturation; $P_{\mathrm{CO}_{2}}$ : carbon dioxide tension. ${ }^{\#}: \mathrm{n}=10 ;{ }^{\natural}: \mathrm{n}=24$.

In an observational study of patients with steroid-responsive nephrotic syndrome, in which PSG was performed and total body water was measured prior to and following treatment with steroids, TANG et al. [24] demonstrated that total body extracellular water decreased from $20.7 \pm 5.9$ to $16.1 \pm 3.1 \mathrm{~L}(\mathrm{p}<0.001)$ in association with a $50 \%$ reduction in the AHI from $34.8 \pm 7.6$ to $16.5 \pm 4.0(\mathrm{p}<0.05)$ in 11 subjects with OSA. The same authors showed in 24 ESRD patients that the AHI increased four-fold (from 3.4 to 14.0 events.h $\mathrm{h}^{-1}$; $\mathrm{p}<0.001$ ) after conversion from nocturnal to continuous ambulatory peritoneal dialysis, in association with $1.47 \mathrm{~L}$ less fluid removal during the latter at night, but without any significant difference in blood urea or creatinine levels [25]. In another study, conversion from conventional to nocturnal haemodialysis led to a reduction in the mean AHI from $25 \pm 25$ to $8 \pm 8$ events $\cdot h^{-1}$ [13], in association with improved control of both uraemia and total body fluid volume. However, the relative roles of improved uraemic status versus reduced fluid volumes in attenuating sleep apnoea were not determined. To address this, we demonstrated, in 15 subjects with sleep apnoea, that removal of $2.2 \mathrm{~L}$ of fluid during a single ultrafiltration session led to a $36 \%$ reduction in AHI, in the absence of any changes in uraemic or metabolic status [14]. Furthermore, the degree of reduction in AHI correlated with the degree of reduction in total body ECFV $\left(\mathrm{r}^{2}=0.322, \mathrm{p}=0.027\right)$. The results of the current study complement these findings in that the total body ECFV was much higher in the sleep apnoea than in the no sleep apnoea group, and total body ECFV was the only independent correlate of the AHI. Furthermore, while elevated BMI is an important risk factor for OSA in the general population [26], in the present study, BMI was within the normal range for the majority of subjects, did not differ between the groups and did not correlate with the AHI. Taken together, these results indicate that fluid overload

FIGURE 2 Total body extracellular fluid volume (ECFV) in the no sleep apnoea and sleep apnoea groups. In the no sleep apnoea group the mean \pm SD total body ECFV was $15.1 \pm 2.6 \mathrm{~L}$ and in the sleep apnoea group it was $17.7 \pm 2.9 \mathrm{~L}$.

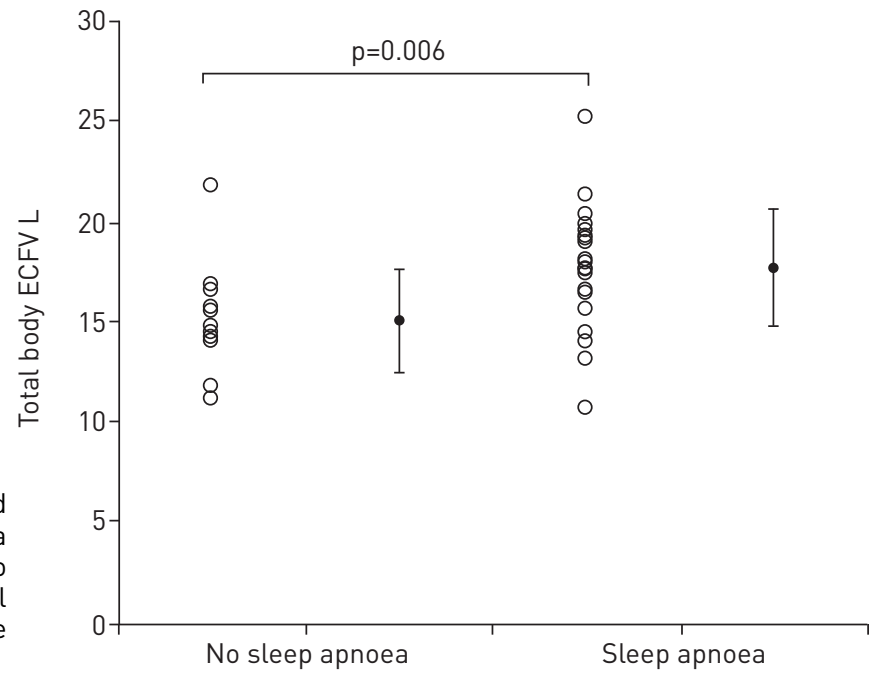


TABLE 4 Fluid volume measurements

\begin{tabular}{|c|c|c|c|}
\hline & $\begin{array}{c}\text { No sleep apnoea } \\
\qquad A \mathrm{HI}<15\end{array}$ & $\begin{array}{c}\text { Sleep apnoea } \\
A H I \geqslant 15\end{array}$ & p-value \\
\hline Subjects & 14 & 28 & \\
\hline Total body fluid volume $L$ & $34.6 \pm 5.8$ & $39.7 \pm 7.7$ & 0.027 \\
\hline Total body ICFV L & $19.5 \pm 3.8$ & $21.9 \pm 5.4$ & 0.177 \\
\hline Total body ECFV index $L \cdot \mathrm{m}^{-2}$ & $8.8 \pm 1.0$ & $9.7 \pm 1.1$ & 0.004 \\
\hline Evening LECFV mL & $1882 \pm 332$ & $2240 \pm 441$ & 0.010 \\
\hline Evening LECFV index $\mathrm{mL} \cdot \mathrm{m}^{-2}$ & $1086 \pm 178$ & $1234 \pm 185$ & 0.020 \\
\hline Overnight change in LECFV $\mathrm{mL}$ & $-210 \pm 75$ & $-272 \pm 117$ & 0.048 \\
\hline Evening TECFV $\mathrm{mL}$ & $2229 \pm 576$ & $2817 \pm 1017$ & 0.028 \\
\hline Evening TECFV index $\mathrm{mL} \cdot \mathrm{m}^{-2}$ & $1184 \pm 465$ & $1554 \pm 524$ & 0.033 \\
\hline Overnight change in TECFV $\mathrm{mL}$ & $157 \pm 179$ & $276 \pm 177$ & 0.059 \\
\hline Evening NECFV mL & $383 \pm 94$ & $467 \pm 102$ & 0.016 \\
\hline Evening NECFV index $\mathrm{mL} \cdot \mathrm{m}^{-2}$ & $222 \pm 48$ & $261 \pm 51$ & 0.031 \\
\hline Overnight change in NECFV $\mathrm{mL}$ & $24 \pm 49$ & $38 \pm 53$ & 0.430 \\
\hline
\end{tabular}

Data are presented as $\mathrm{n}$ or mean $\pm \mathrm{SD}$, unless otherwise stated. $\mathrm{AHI}$ : apnoea-hypopnoea index; ICFV: intracellular fluid volume; ECFV: extracellular fluid volume; LECFV: leg extracellular fluid volume; TECFV: thoracic extracellular fluid volume; NECFV: neck extracellular fluid volume.

contributes to the pathogenesis of both OSA and CSA in ESRD patients, and that fluid removal is a mechanism by which sleep apnoea can be improved in this population.

The exact mechanisms by which fluid overload contributes to the pathogenesis of sleep apnoea have yet to be fully elucidated. Yumino et al. [16] showed that the degree of overnight change in leg fluid volume correlated with the AHI in heart failure patients with either OSA or CSA, suggesting that nocturnal rostral fluid shift is a unifying mechanism in the pathogenesis of both OSA and CSA in patients with heart failure. At that time, technical limitations did not allow for measurement of NECFV and TECFV. However, in the OSA subjects, there was an increase in neck circumference overnight that correlated with the AHI, suggesting that fluid shift from the leg was redistributed to the neck. Conversely, in the CSA subjects, the overnight change in LECFV correlated inversely with the mean transcutaneous carbon dioxide tension $\left(\mathrm{PCO}_{2}\right)$ during sleep, which in turn was inversely related to the AHI. These observations suggested that some of the fluid shifting from the legs redistributed to the chest, leading to increased pulmonary congestion with subsequent stimulation of pulmonary irritant receptors, leading to hyperventilation and subsequent lowering of $\mathrm{PCO}_{2}$ [27]. This could lead to CSA if the $\mathrm{PCO}_{2}$ during sleep fell below the apnoea threshold [27]. Given that ESRD patients are fluid overloaded, it is plausible that increases in TECFV could also contribute to the pathogenesis of CSA in ESRD patients by the same mechanisms.

With regards to the pathogenesis of OSA in ESRD, in a study of 26 ESRD patients, 12 of whom had OSA, the degree of overnight fluid shift from the legs did not correlate with the AHI, but did correlate with the apnoea-hypopnoea time, an alternative marker of sleep apnoea severity [15]. ELIAS et al. [28] subsequently showed, in 20 ESRD patients, that the only significant independent correlates of OSA severity, as characterised by the AHI, were internal jugular vein volume and upper airway mucosal water content, measured by magnetic resonance imaging. These observations suggested that increased intravascular and interstitial fluid accumulation in the neck tissues surrounding the upper airway could lead to increased collapsibility of the upper airway. In the current study, the finding of a higher mean baseline NECFV in the sleep apnoea compared to the no sleep apnoea group is consistent with those previous finding [28]. Consistent with those results, we also found no significant difference in the overnight change in NECFV between the groups. This suggests the possibility that in ESRD patients the magnitude of fluid overload is

TABLE 5 Multiple logistic regression analysis, with presence of sleep apnoea as dependent factor

\begin{tabular}{lcc} 
& Odds ratio $(95 \%$ Cl) & p-value \\
\hline Age & $1.161(1.046-1.288)$ & 0.005 \\
Total body ECFV index & $3.043(1.016-9.113)$ & 0.047 \\
\hline
\end{tabular}

ECFV: extracellular fluid volume. 
such that it leads to increased NECFV regardless of body position, and therefore NECFV does not subsequently increase overnight any more in those with sleep apnoea than in those without it.

Studies in subjects with normal renal function and in patients with ESRD suggest that ventilatory control system instability and increased chemosensitivity may play a role in the pathogenesis of CSA and OSA [29, 30]. In this context, the finding of a significantly higher TECFV in the sleep apnoea compared to the no sleep apnoea group is of particular interest. As described above, hypocapnia, secondary to pulmonary congestion, may predispose to ventilatory instability and CSA through mechanisms described previously in heart failure patients $[27,31]$. With respect to OSA, the subsequent periodic breathing, with waxing and waning of the respiratory drive, could lead to reductions in the neural drive to upper airway dilator muscles and, in susceptible individuals, lead to recurrent upper airway collapse and OSA. While there was no difference in the mean transcutaneous $\mathrm{PCO}_{2}$ between the groups in our study, this does not necessarily exclude the possibility that the relatively low mean $\mathrm{PCO}_{2}$ of $39.5 \mathrm{mmHg}$ in the sleep apnoea group could have led to ventilatory instability, at least in some patients. Indeed, we have previously shown that following ultrafiltration there was a mean reduction in TECFV of $450 \mathrm{~mL}$ that was accompanied by an increase in the overnight transcutaneous $\mathrm{PCO}_{2}$ from $39.2 \pm 1.9$ to $42.3 \pm 5.1 \mathrm{mmHg}(\mathrm{p}=0.042)$ and by attenuation of sleep apnoea [14]. These findings suggested that fluid removal and the subsequent reduction in TECFV led to a reduction in respiratory drive, increased ventilatory stability and attenuation of both OSA and CSA. In this regard, further research is needed to investigate the relative contributions of increased neck, chest and leg fluid volumes as well as to determine the relative roles of upper airway mechanics and respiratory control system stability in the pathogenesis of both OSA and CSA in ESRD patients.

One of the limitations of this study is that the sleep apnoea group was older and had a higher proportion of males than the no sleep apnoea group, characteristics that would increase the risk for OSA [2, 32]. However, we showed in both multiple logistic regression and linear regression analyses, that included age, sex and BMI, that total body ECFV index remained independently associated with both the presence of sleep apnoea and the AHI, which supports an important role for fluid in the pathogenesis of sleep apnoea in ESRD. Another limitation is that, in presenting the results of fluid volume measurements, we did not divide the sleep apnoea group into those with OSA and those with CSA. This was due in part to the small number of patients with CSA $(n=7)$, but also because we did not see any difference in total body or segmental fluid volumes between those patients with OSA or CSA. Accordingly, and given the lack of any intervention in this study, it was not possible to determine a cause-effect relationship regarding the role of NECFV and TECFV in the pathogenesis of sleep apnoea in ESRD.

In conclusion, among patients with ESRD, we showed that subjects with sleep apnoea had significantly higher total body ECF volume and segmental fluid volumes than those with no sleep apnoea. These results complement our previous findings that fluid removal by ultrafiltration reduces the AHI of ESRD patients with either OSA or CSA in proportion to the reduction in total body ECFV. Taken together, these results support the role of fluid overload as an important mechanism in the pathogenesis of sleep apnoea in ESRD. They indicate the need for further research to determine the relationship between increased neck and thoracic fluid volumes, and upper airway mechanics and respiratory control system stability, respectively. Ultimately, studies will be required to assess the effects of more aggressive fluid removal on a chronic basis on sleep apnoea severity in ESRD patients.

\section{Acknowledgements}

The authors wish to thank Celine d'Gama, Rose Faratro, Stella Fung and Elizabeth Wong (Home Hemodialysis Unit, Toronto General Hospital, Toronto, ON, Canada) for their invaluable assistance with subject recruitment.

\section{References}

1 Lyons OD, Bradley TD, Chan CT. Hypervolemia and sleep apnea in kidney disease. Semin Nephrol 2015; 35: 373-382.

2 Peppard PE, Young T, Barnet JH, et al. Increased prevalence of sleep-disordered breathing in adults. Am J Epidemiol 2013; 177: 1006-1014.

3 Kraus MA, Hamburger RJ. Sleep apnea in renal failure. Adv Perit Dial 1997; 13: 88-92.

4 Kimmel PL, Miller G, Mendelson WB. Sleep apnea syndrome in chronic renal disease. Am J Med 1989; 86: 308-314.

5 Wadhwa NK, Seliger M, Greenberg HE, et al. Sleep related respiratory disorders in end-stage renal disease patients on peritoneal dialysis. Perit Dial Int 1992; 12: 51-56.

6 Unruh ML, Sanders MH, Redline S, et al. Sleep apnea in patients on conventional thrice-weekly hemodialysis: comparison with matched controls from the Sleep Heart Health Study. J Am Soc Nephrol 2006; 17: 3503-3509.

7 Yumino D, Wang H, Floras JS, et al. Prevalence and physiological predictors of sleep apnea in patients with heart failure and systolic dysfunction. J Card Fail 2009; 15: 279-285.

8 Tada T, Kusano KF, Ogawa A, et al. The predictors of central and obstructive sleep apnoea in haemodialysis patients. Nephrol Dial Transplant 2007; 22: 1190-1197.

9 Beecroft JM, Pierratos A, Hanly PJ. Clinical presentation of obstructive sleep apnea in patients with end-stage renal disease. J Clin Sleep Med 2009; 5: 115-121. 
10 Arzt M, Young T, Finn L, et al. Sleepiness and sleep in patients with both systolic heart failure and obstructive sleep apnea. Arch Intern Med 2006; 166: 1716-1722.

11 Kalantar-Zadeh K, Regidor DL, Kovesdy CP, et al. Fluid retention is associated with cardiovascular mortality in patients undergoing long-term hemodialysis. Circulation 2009; 119: 671-679.

12 Tang SC, Lam B, Yao TJ, et al. Sleep apnea is a novel risk predictor of cardiovascular morbidity and death in patients receiving peritoneal dialysis. Kidney Int 2010; 77: 1031-1038.

13 Hanly PJ, Pierratos A. Improvement of sleep apnea in patients with chronic renal failure who undergo nocturnal hemodialysis. N Engl J Med 2001; 344: 102-107.

14 Lyons OD, Chan CT, Yadollahi A, et al. Effect of ultrafiltration on sleep apnea and sleep structure in patients with end-stage renal disease. Am J Respir Crit Care Med 2015; 191: 1287-1294.

15 Yadollahi A, Gabriel JM, White LH, et al. A randomized, double crossover study to investigate the influence of saline infusion on sleep apnea severity in men. Sleep 2014; 37: 1699-1705.

16 Yumino D, Redolfi S, Ruttanaumpawan P, et al. Nocturnal rostral fluid shift: a unifying concept for the pathogenesis of obstructive and central sleep apnea in men with heart failure. Circulation 2010; 121: 1598-1605.

17 Elias RM, Bradley TD, Kasai T, et al. Rostral overnight fluid shift in end-stage renal disease: relationship with obstructive sleep apnea. Nephrol Dial Transplant 2012; 27: 1569-1573.

18 Redolfi S, Yumino D, Ruttanaumpawan P, et al. Relationship between overnight rostral fluid shift and obstructive sleep apnea in nonobese men. Am J Respir Crit Care Med 2009; 179: 241-246.

19 Friedman O, Bradley TD, Chan CT, et al. Relationship between overnight rostral fluid shift and obstructive sleep apnea in drug-resistant hypertension. Hypertension 2010; 56: 1077-1082.

20 Rechtschaffen A, Kales A. A Manual of Standardized Terminology, Techniques and Scoring System for Sleep Stages of Human Subjects. Los Angeles, UCLA Brain Information Service/Brain Research Institute, 1968

21 Berry RB, Gamaldo CE, Harding SM, et al. The AASM Manual for the Scoring of Sleep and Associated Events: Rules, Terminology and Technical Specifications. Version 2. Darien, American Academy of Sleep Medicine, 2012.

22 Kyle UG, Bosaeus I, De Lorenzo AD, et al. Bioelectrical impedance analysis - part II: utilization in clinical practice. Clin Nutr 2004; 23: 1430-1453.

23 Yadollahi A, Singh B, Bradley TD. Investigating the dynamics of supine fluid redistribution within multiple body segments between men and women. Ann Biomed Eng 2015; 43: 2131-2142.

24 Tang SC, Lam B, Lam JC, et al. Impact of nephrotic edema of the lower limbs on obstructive sleep apnea: gathering a unifying concept for the pathogenetic role of nocturnal rostral fluid shift. Nephrol Dial Transplant 2012; 27: 2788-2794

25 Tang SC, Lam B, Ku PP, et al. Alleviation of sleep apnea in patients with chronic renal failure by nocturnal cycler-assisted peritoneal dialysis compared with conventional continuous ambulatory peritoneal dialysis. J Am Soc Nephrol 2006; 17: 2607-2616.

26 Tishler PV, Larkin EK, Schluchter MD, et al. Incidence of sleep-disordered breathing in an urban adult population: the relative importance of risk factors in the development of sleep-disordered breathing. JAMA 2003; 289: 2230-2237.

27 Naughton M, Benard D, Tam A, et al. Role of hyperventilation in the pathogenesis of central sleep apneas in patients with congestive heart failure. Am Rev Respir Dis 1993; 148: 330-338.

28 Elias RM, Chan CT, Paul N, et al. Relationship of pharyngeal water content and jugular volume with severity of obstructive sleep apnea in renal failure. Nephrol Dial Transplant 2013; 28: 937-944.

29 Wellman A, Jordan AS, Malhotra A, et al. Ventilatory control and airway anatomy in obstructive sleep apnea. Am J Respir Crit Care Med 2004; 170: 1225-1232.

30 Beecroft J, Duffin J, Pierratos A, et al. Enhanced chemo-responsiveness in patients with sleep apnoea and end-stage renal disease. Eur Respir J 2006; 28: 151-158.

31 Sin DD, Fitzgerald F, Parker JD, et al. Risk factors for central and obstructive sleep apnea in 450 men and women with congestive heart failure. Am J Respir Crit Care Med 1999; 160: 1101-1106.

32 Edwards BA, O'Driscoll DM, Ali A, et al. Aging and sleep: physiology and pathophysiology. Semin Respir Crit Care Med 2010; 31: 618-633. 